\title{
Intracellular Glucose Oxidation and Glycogen Synthase Activity Are Reduced in Non-insulin-dependent (Type II) Diabetes Independent of Impaired Glucose Uptake
}

Anne W. Thorburn, Barry Gumbiner, Fred Bulacan, Penny Wallace, and Robert R. Henry

Department of Medicine, University of California, San Diego and the Metabolism Division,

Veterans Administration Medical Center, San Diego, California 92161

\begin{abstract}
To examine whether reduced rates of oxidative $\left(G_{o x}\right)$ and nonoxidative $\left(\mathbf{N}_{\mathrm{ox}}\right)$ glucose metabolism in non-insulin-dependent diabetes mellitus (NIDDM) are due to reduced glucose uptake, intrinsic defects in intracellular glucose metabolism or increased fat oxidation $\left(F_{o x}\right)$, indirect calorimetry was performed at similar glucose uptake rates in eight nonobese NIDDM and eight comparable nondiabetic subjects. Three glucose clamp studies were performed: one in the nondiabetic and two in the NIDDM subjects. In the nondiabetic subjects, glucose uptake was increased to $7.62 \pm 0.62 \mathrm{mg} / \mathrm{kg}$ of fat-free mass (FFM) per min by increasing serum insulin to $309 \mathrm{pmol} /$ liter at a glucose concentration of $5.1 \mathrm{mmol} / \mathrm{liter}$. By raising the concentration of either serum glucose or insulin fourfold in the NIDDM subjects, glucose uptake was matched to nondiabetic subjects $(8.62 \pm 0.49$ and $8.59 \pm 0.51 \mathrm{mg} / \mathrm{kg}$ FFM per $\mathrm{min}$, respectively, $P=$ NS). Skeletal muscle glycogen synthase activity and plasma lactate levels were measured to characterize $\mathbf{N}_{\mathbf{o x}}$.

When glucose uptake was matched to nondiabetics by hyperglycemia or hyperinsulinemia, $G_{o x}$ was reduced by $26-28 \%$ in NIDDM $(P<0.025)$ whereas $F_{\text {ox }}$ was similar. $N_{o x}$ was greater in NIDDM $(P<0.01)$ and was accompanied by increases in circulating lactate levels. Glycogen synthase activity was reduced by $41 \%(P<0.025)$ when glucose uptake was matched by hyperglycemia. Glycogen synthase activity was normalized in NIDDM, however, when glucose uptake was matched by hyperinsulinemia.

Therefore, a defect in $G_{0 x}$ exists in nonobese NIDDM subjects which cannot be overcome by increasing glucose uptake or insulin. Since both glucose uptake and $F_{\text {ox }}$ were similar in the two subject groups these factors were not responsible for reduced $G_{o x}$. Increased $N_{o x}$ in NIDDM is primarily into lactate. Reduced glycogen synthase activity in NIDDM is independent of glucose uptake but can be overcome by increasing the insulin concentration. (J. Clin. Invest. 1990. 85:522-529.) glucose oxidation • glycogen synthase $\bullet$ non-insulin-dependent diabetes mellitus
\end{abstract}

\section{Introduction}

Under normal circumstances insulin stimulates peripheral tissues, most notably skeletal muscle (1), to take up glucose

Address reprint requests to Dr. Henry, Veterans Administration Medical Center (V111G), 3350 La Jolla Village Drive, San Diego, CA 92161.

Received for publication 19 June 1989 and in revised form 8 September 1989.

The Journal of Clinical Investigation, Inc.

Volume 85, February 1990, 522-529 which is then metabolized by either oxidative or nonoxidative pathways. In non-insulin-dependent diabetes mellitus (NIDDM), ${ }^{1}$ peripheral insulin resistance is a characteristic feature which impairs insulin's ability to stimulate glucose uptake and is associated with multiple abnormalities in intracellular glucose metabolism. Although defects in both oxidative and nonoxidative glucose metabolism have been demonstrated in NIDDM (2-7), current evidence indicates that the nonoxidative pathways are more significantly affected. This conclusion is based on the close correlations which have been demonstrated between defects in glucose uptake and nonoxidative glucose metabolism (7-10). Additional correlations between glucose uptake and the activity of the rate-limiting enzyme for glycogen synthesis (glycogen synthase) $(7,10,11)$, plus the similarity in the sensitivity of glucose uptake and glycogen synthase for insulin (12) have led several investigators to further speculate that defects in the nonoxidative glycogen synthesis pathway may be the cause of the reduced rates of glucose uptake present in NIDDM and other states of insulin resistance $(7,10-15)$.

The conclusions drawn from these studies can be questioned, however, for the following reasons. First, reduced rates of intracellular glucose metabolism may be a consequence rather than a cause of reduced rates of tissue glucose uptake. That is, because of impaired insulin-mediated glucose uptake, less intracellular glucose than normal is available to traverse these metabolic pathways in NIDDM. Alternatively, since both glucose uptake and glycogen synthase can be stimulated by insulin, a simple correlation between these two processes does not necessarily indicate a cause and effect relationship. In support of this notion, there is some experimental evidence (16-20) that defects in insulin's effects on intracellular glucose metabolism may exist independent of the defect in insulinmediated glucose uptake.

To investigate whether the reduced rates of oxidative and nonoxidative glucose metabolism previously found in NIDDM are the result of reduced rates of glucose uptake or intrinsic defects in intracellular glucose metabolism, we carried out comparative studies in eight nonobese male subjects with and without NIDDM. Rates of oxidative and nonoxidative glucose metabolism were measured using indirect calorimetry in the basal state and at the same rate of glucose uptake in both groups. Glucose uptake was increased in the NIDDM subjects by increasing either the serum glucose or insulin fourfold that of nondiabetic control subjects using the combined pancreatic-glucose clamp technique. Assuming that glycogen

1. Abbreviations used in this paper: FFM, fat-free mass: GU, glucose uptake; G6P, glucose 6-phosphate; HGO, hepatic glucose output; NIDDM, non-insulin-dependent diabetes mellitus; PDH, pyruvate dehydrogenase; UDPG, uridine 5'-diphosphate glucose. 
synthase is the major determinant of glycogen deposition in muscle (7), we estimated the contribution of glycogen synthesis to nonoxidative glucose metabolism by measuring glycogen synthase activity in biopsies of skeletal muscle in all subjects at the end of the basal and glucose clamp studies. Circulating plasma lactate levels were also measured to further characterize the composition of $\mathrm{N}_{\mathrm{ox}}$. Fat oxidation was also measured to examine whether defects in glucose oxidation might be the result of increased fat oxidation as previously reported in $\operatorname{NIDDM}(21,22)$.

\section{Methods}

Subjects. Eight male nonobese subjects with NIDDM and eight male nonobese control subjects with normal glucose tolerance (23) participated in the studies. The clinical and metabolic characteristics of the subjects are listed in Table I. Age, weight, fat-free mass (FFM, 24), and body mass index did not differ in the NIDDM and control subjects. Fasting serum glucose in the NIDDM subjects was twice that of the controls $(P<0.005)$; fasting serum insulin was $78 \%$ greater $(P<0.05)$. The NIDDM subjects were healthy, except for diabetes, and did not have significant diabetic complications or hypertension. Five subjects were on sulfonylurea therapy, one was on insulin, one was on diet therapy only, and one had just been diagnosed and not yet treated. Oral hypoglycemic agents and insulin were withdrawn at least 2 wk before the studies. No subject was taking any other medication known to affect carbohydrate metabolism. Duration of overt diabetes was $10 \pm 3$ yr (mean \pm SEM). Fasting glycosylated hemoglobin in the NIDDM subjects was $9.4 \pm 1.2 \%$. The control subjects were screened to ensure they were healthy and had no family history of diabetes. Written informed consent was obtained from each subject. The experimental protocol was approved by the Committee on Human Investigation of the University of California, San Diego.

Study protocol. Subjects were admitted to the Special Diagnostic and Treatment Unit of the Veterans Administration Medical Center in San Diego and consumed a weight-maintenance standardized solid diet containing $55 \%$ of calories as carbohydrate, $30 \%$ as fat, and $15 \%$ as protein for at least $24 \mathrm{~h}$ before each study.

Three studies were performed, one in the control subjects and two in the NIDDM subjects. Each study consisted of a basal (i.e., postabsorptive) period followed by a glucose clamp. As shown in Table II, the same rate of glucose uptake was achieved during all three glucose clamps by raising either the glucose level (designated clamp " $G$ ") or the insulin level (clamp "I") in the NIDDM subjects approximately fourfold above the levels used in the control subjects.

Each study was performed after a 12-14 h overnight fast. On the

Table I. Clinical and Metabolic Characteristics*

\begin{tabular}{lcc}
\hline & Control subjects & NIDDM subjects \\
\hline Age $(y r)$ & $49 \pm 6$ & $59 \pm 3$ \\
Weight $(\mathrm{kg})$ & $75.6 \pm 3.4$ & $78.8 \pm 5.0$ \\
Fat-free mass $(\mathrm{kg})^{\ddagger}$ & $57.7 \pm 2.0$ & $60.5 \pm 3.8$ \\
Body mass index $\left(\mathrm{kg} / \mathrm{m}^{2}\right)$ & $25.0 \pm 0.7$ & $25.4 \pm 1.1$ \\
Fasting serum glucose $(\mathrm{mmol} / \mathrm{liter})$ & $5.0 \pm 0.1$ & $9.9 \pm 1.1^{\S}$ \\
Fasting serum insulin $(\mathrm{pmol} / \mathrm{liter})$ & $36 \pm 7$ & $64 \pm 14^{\| 1}$ \\
\hline
\end{tabular}

* Data expressed as mean \pm SEM.

${ }^{\ddagger}$ Determined by underwater weighing with correction for residual lung volume (24).

$\$ P<0.005$ from control subjects.

" $P<0.05$ from control subjects.
Table II. Clamp Study Design to Achieve the Same Rate of Glucose Uptake of 7-9 $\mathrm{mg} / \mathrm{kg}$ FFM per min in the Control and NIDDM Subjects

\begin{tabular}{lcc}
\hline & Insulin & Glucose \\
\hline & pmol/m ${\text { per } \text { min }^{*}}^{*}$ & mmol/liter \\
Control subjects & 150 & 5 \\
NIDDM subjects & & \\
Clamp G & 150 & $15-30$ \\
Clamp I & $450-900$ & 5 \\
\hline
\end{tabular}

$* 1 \mathrm{pmol} / \mathrm{m}^{2}$ per $\mathrm{min}=0.14 \mathrm{mU} / \mathrm{m}^{2}$ per $\mathrm{min}$.

$\neq 1 \mathrm{mmol} /$ liter $=18 \mathrm{mg} / \mathrm{dl}$.

morning of each test two peripheral venous catheters were inserted: one in an antecubital vein for infusates, the other in a retrograde fashion into a dorsal vein of the ipsilateral hand for blood sampling. The hand was kept in a warming device at $60-70^{\circ} \mathrm{C}$ to achieve arterialization of venous blood for sampling (25). Each experiment lasted an average of $7.5 \mathrm{~h}$ : a 2.5 -h basal period followed by a 5 -h clamp. A summary of the study procedures is given in Fig. $1 .\left[3-{ }^{3} \mathrm{H}\right]$ glucose was infused during the entire study so that rates of glucose uptake could be isotopically determined. The clamp was started with an intravenous infusion of insulin (crystalline human insulin [Humulin R], Eli Lilly and Co., Indianapolis, IN) as a bolus delivered in a logarithmically decreasing pattern followed by a continuous rate of insulin infusion (as listed in Table II). Glucose was clamped by a variable infusion of $20 \%$ glucose, at a rate determined in a feedback fashion by the measurement of serum glucose at 5-10-min intervals (26). Somatostatin $(0.08$ $\mathrm{pmol} / \mathrm{kg}$ per min, cyclic form, Bachem Inc., Torrence, CA) was infused in all clamp studies to suppress endogenous insulin secretion. $\mathrm{KCl}$ and $\mathrm{K}_{2} \mathrm{PO}_{4}$ were also infused at a rate of $0.16 \mathrm{mmol} / \mathrm{min}$ to maintain the serum potassium level between 3.5 and $4.5 \mathrm{mmol} / \mathrm{liter}$ and thus avoid any potential deleterious effects of hypokalemia. During the last 30 min of the basal and clamp periods, steady-state measurements were made of rates of glucose uptake, hepatic glucose output, glucose oxidation, fat oxidation, and protein oxidation as well as levels of serum glucose and insulin, and plasma levels of free fatty acids and lactate. In addition, glycogen synthase activity was determined on needle biopsy specimens of vastus lateralis muscle obtained at the end of a basal period and at completion of each clamp study. The basal muscle biopsy was collected on a separate day so that only one biopsy was performed per day. These methods are discussed in detail in the following sections.

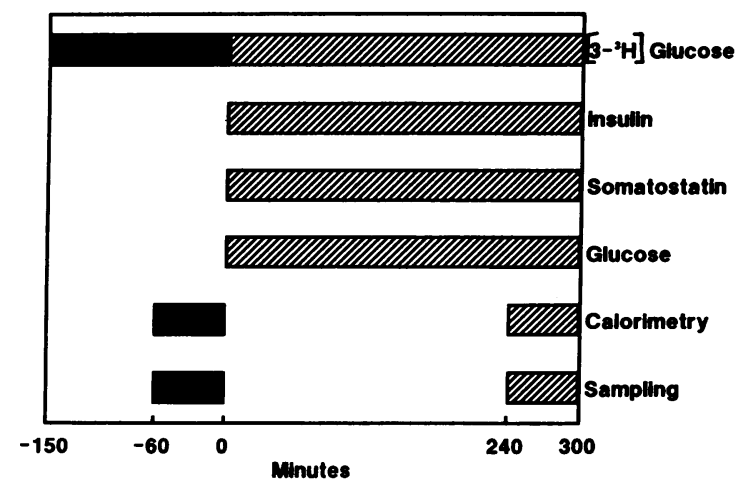

Figure 1. Schema of the study design. Procedures performed during the 150 -min basal period are illustrated by the solid black bars; those performed during the 300 -min clamp are illustrated by the hatched bars. 
Measurement of glucose uptake and hepatic glucose output. The rate of glucose appearance $(\mathrm{Ra})$ and disappearance $(\mathrm{Rd})$ was quantitated in the basal state and during the glucose clamp by infusing [3${ }^{3} \mathrm{H}$ ]glucose in a primed continuous manner (27). With this technique, $45 \mu \mathrm{Ci}$ of tracer was injected as a bolus dose, followed by a continuous infusion at a rate of $0.60 \mu \mathrm{Ci} / \mathrm{min}$ throughout the study period. The tracer was allowed to equilibrate for $120 \mathrm{~min}$, and glucose concentration and specific activity were measured for the subsequent 30 -min period at 10-min intervals. $\mathrm{Ra}$ and $\mathrm{Rd}$ were calculated in the basal state using the Steele equation assuming steady state conditions (28) and taking into account any urinary glucose loss in the NIDDM subjects. The rate of basal hepatic glucose output (HGO) was determined from $\mathrm{Ra}$ since, in the basal state, essentially all endogenous glucose is produced by the liver. During the last hour of the 5-h insulin infusion, blood was collected at 20-min intervals to analyze the glucose concentration and specific activity. Ra and Rd were calculated with the Steele equations in their modified derivative form, since the tracer exhibits non-steady-state kinetics under these conditions (29). The glucose uptake rate (in milligrams per kilogram of FFM per minute) during the last $30 \mathrm{~min}$ of the clamp was calculated from the glucose infusion rate, corrected for changes in glucose pool size and any urinary glucose loss plus any residual HGO. Residual HGO was calculated by subtracting the rate of exogenous glucose infused from the isotopically measured $\mathrm{Ra}$. If the glucose infusion rate was greater than $\mathrm{Ra}, \mathrm{HGO}$ was negative and assumed to be $100 \%$ suppressed. In the NIDDM subjects HGO was only $83 \%$ suppressed in the hyperglycemic clamp and $92 \%$ suppressed in the hyperinsulinemic clamp compared with $100 \%$ in the control subjects. This isotope dilution technique has been criticized recently because it tends to underestimate $\mathrm{Ra}$, probably owing to modeling error and/or isotope impurity (30), and may be significant at high turnover rates in non-steady-state conditions. In the present study, however, significant underestimates are unlikely to have occurred since the calculation of glucose turnover rates would be largely modelindependent under the virtual steady-state conditions that we used. In addition, tracer impurity is unlikely since the tracer used was $>99 \%$ pure and no difference in arteriovenous $\left[3-{ }^{3} \mathrm{H}\right]$ glucose specific activity across the leg has been shown in our laboratory at high glucose turnover rates $(31)$.

Indirect calorimetry: determination of glucose, fat and protein oxidation. Respiratory exchange measurements were made during the last hour of both the basal and clamp periods using an open-circuit indirect calorimeter. A constant fraction of the air expired by the subject from a transparent ventilated hood was desaturated, filtered, and analyzed for oxygen $\left(\mathrm{O}_{2}\right)$ and carbon dioxide $\left(\mathrm{CO}_{2}\right) \cdot \mathrm{O}_{2}$ was measured by a magnetopneumatic Magnos $4 \mathrm{G}$ and $\mathrm{CO}_{2}$ by an infrared Uras 3G (Hartmann and Braun, Frankfurt, FRG). Standard gas mixtures of $20.00 \% \mathrm{O}_{2}$ and $1.00 \% \mathrm{CO}_{2}$ were used to calibrate the analyzers before and after each study. Acetone, which has an respiratory quotient $\left(\mathrm{CO}_{2}\right.$ production $/ \mathrm{O}_{2}$ consumption) of 0.75 , was burned in a container inside the hood before and after each study to check the complete system of indirect calorimetry. Before and after the subject was removed from the headbox, blood was collected to measure urea, and urine was collected for analysis of creatinine, urea, and uric acid. After correcting for changes in urea clearance (32), the nitrogen in these three compounds was combined to give total urinary nitrogen excretion $(\mathrm{N}$, in grams per minute). This was used to calculate the rate of protein oxidation $\left(\mathrm{P}_{\mathrm{ox}}\right)$ where $P_{o x}=6.25 \times \mathrm{N}$. From the mean rates of $\mathrm{O}_{2}$ consumed $\left(\mathrm{VO}_{2}\right.$, in liters per minute) and $\mathrm{CO}_{2}$ produced $\left(\mathrm{VCO}_{2}\right)$ the rates of glucose $\left(\mathrm{G}_{\mathrm{ox}}\right)$ and fat $\left(\mathrm{F}_{\mathrm{ox}}\right)$ oxidized in milligrams per kilogram of FFM per minute were calculated using the following equations:

$\mathrm{G}_{\mathrm{ox}}=\left[\left(4.54 \mathrm{VCO}_{2}-3.20 \mathrm{VO}_{2}-2.87 \mathrm{~N}\right) / \mathrm{FFM}\right] \times 1,000$.

$F_{o x}=\left[\left(-1.67 \mathrm{VCO}_{2}+1.67 \mathrm{VO}_{2}-1.92 \mathrm{~N}\right) / \mathrm{FFM}\right] \times 1,000$.

Since approximately $72 \%$ of the plasma glucose turnover is derived from glycogen in the basal state (33), rates of basal Rd were expressed as milligrams of glycosyl units per kilogram FFM per minute and the
$\mathrm{VCO}_{2}$ and $\mathrm{VO}_{2}$ of glycogen instead of glucose were used to calculate basal carbohydrate oxidation $\left(C_{o x}\right)(34)$, i.e.,

$\mathrm{C}_{\mathrm{ox}}=\left[\left(4.09 \mathrm{VCO}_{2}-2.88 \mathrm{VO}_{2}-2.58 \mathrm{~N}\right) / \mathrm{FFM}\right] \times 1,000$.

For simplicity, however, the phrase "glucose oxidation" is used to refer to carbohydrate oxidation in the basal state. Nonoxidative glucose metabolism was determined by the difference between the rates of glucose uptake and glucose oxidation.

Analytical methods. Blood drawn for glucose was immediately separated by an Eppendorf microcentrifuge (Brinkmann Instruments Co., Inc., Westbury, NY) and serum glucose determined by the glucose oxidase method (model 23A, Yellow Springs Instrument Co., Yellow Springs, $\mathrm{OH}$ ). Blood for serum insulin was collected in untreated tubes and allowed to clot at room temperature before the supernatant was removed and frozen at $-20^{\circ} \mathrm{C}$. Insulin was measured by a specific double-antibody radioimmunoassay (35). Blood for analysis of lactate and glucose specific activity was collected in tubes containing potassium oxalate plus sodium fluoride. Blood for analysis of free fatty acids was collected in tubes containing $100 \mu \mathrm{l}$ of EDTA. These samples were immediately placed on ice until centrifuged then stored at $-20^{\circ} \mathrm{C}$ until assayed. Plasma free fatty acids were measured by the colorimetric method of Itaya and Vi (36). Plasma L-lactate was determined by the lactate oxidase method using a 23L-Lactate Analyzer (Yellow Springs Instrument Co.). Urea nitrogen, creatinine, and uric acid were assessed in serum and urine using routine, semiautomated methods (Hitachi 737, Boehringer Mannheim Diagnostics, Inc., Indianapolis, IN). Total glycosylated hemoglobin was determined on freshly drawn whole blood using the Isolab Glyc-Afin GHb test kit (Isolab, Akron, OH). The normal reference range for this assay was $4.1-8.1 \%$.

Glycogen synthase determination. At the end of a basal period and each clamp study, percutaneous muscle biopsies were obtained on alternating thighs from the vastus lateralis muscle with a 5-mm diam side-cutting needle using a modification of the procedure described by Bergstrom (37). The skin and fascia $12-16 \mathrm{~cm}$ above the patella on the lateral aspect of the thigh was anesthetized with $2-3 \mathrm{ml}$ of $2 \%$ xylocaine without epinephrine and a small incision made through the skin with a scalpel. The biopsy needle was inserted $3-5 \mathrm{~cm}$ beyond the fascia into the muscle and three rapid passes made to yield muscle samples of $50-75 \mathrm{mg}$ in size. The specimen was blotted, frozen in liquid nitrogen within $10 \mathrm{~s}$, and stored in liquid nitrogen until assayed. Glycogen synthase (EC 2.4.1.11) was measured using modifications of the methods of Nuttall et al. (38) and Thomas et al. (39).

Muscle samples $(50 \mathrm{mg})$ were homogenized at $4^{\circ} \mathrm{C}$ with an extraction buffer at pH 7.4 ( $2 \mathrm{mmol} /$ liter of EDTA and 1,4-dithiothreitol, 20 $\mathrm{mmol} /$ liter $\mathrm{NaF}$, and $50 \mathrm{mmol} / \mathrm{liter} \mathrm{KH}_{2} \mathrm{PO}_{4}$ and $\mathrm{K}_{2} \mathrm{HPO}_{4}$ ). After centrifugation $(20,000 \mathrm{~g}$ for $20 \mathrm{~min}), 0.5 \mathrm{ml}$ of the supernatant was added to $2.0 \mathrm{ml}$ of synthase buffer at pH $7.8(20 \mathrm{mmol} / \mathrm{liter}$ of EDTA, $25 \mathrm{mmol} / \mathrm{liter} \mathrm{NaF}$, and $50 \mathrm{mmol} /$ liter Tris- $\mathrm{HCl}$ ). Glycogen synthase was determined in this diluted extract by measuring the incorporation of radioactive $\left[{ }^{14} \mathrm{C}\right]$ glucose from uridine 5 -diphosphate glucose (UDPG) into glycogen. To assay glycogen synthase, $30 \mu$ of the extract was added to $60 \mu \mathrm{l}$ of a reaction mixture consisting of $0.03 \mu \mathrm{Ci}$ of uridine diphospho-D-[U- $\left.-{ }^{14} \mathrm{C}\right]$ glucose (DuPont Co., Wilmington, DE), $1 \%$ glycogen, $0.3 \mathrm{mmol} /$ liter UDPG and a varying concentration of glucose-6-phosphate (G6P, 0-10 mmol/liter). Blank reaction mixture tubes were assayed with boiled extract. Total counts were determined using reaction mixture without extract. After incubating at $30^{\circ} \mathrm{C}$ for 30 min the reaction was stopped by spotting $75 \mu \mathrm{l}$ of each sample onto filter paper and washing three times in $66 \%$ ethanol (for one 45 -min and two 30 -min periods) before drying in acetone. ${ }^{14} \mathrm{C}$ was then counted on a scintillation spectrometer. Protein was also assayed in the extract (40) so that units of synthase activity could be expressed as nanomoles of $\left[{ }^{14} \mathrm{C}\right] U D P G$ incorporated into glycogen per minute per milligram of protein.

Results are expressed as the fractional velocity of glycogen synthase at $0.1 \mathrm{mmol} /$ liter of $\mathrm{G} 6 \mathrm{P}$ (termed $\mathrm{FV}_{0.1}$ ) i.e., the activity of glycogen synthase assayed at $0.1 \mathrm{mmol} /$ liter of G6P divided by the activity at 10 


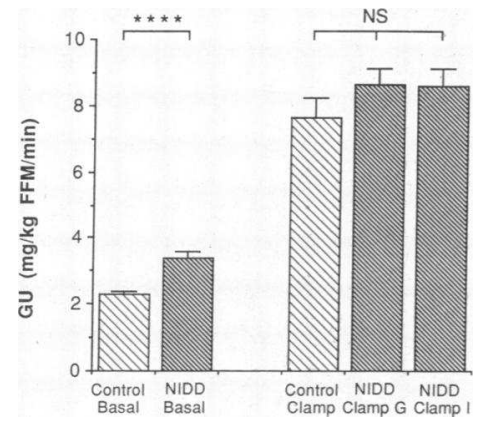

Figure 2. Rates of glucose uptake (GU) in the control and NIDDM subjects under basal and clamp conditions. Basal glucose uptake was significantly higher in the NIDDM subjects $(* * * * P<0.005)$. In the control clamp glucose and insulin concentrations were $5 \mathrm{mmol} / \mathrm{liter}$ and 309 pmol/liter, respectively. In the NIDDM clamps either glucose or insulin levels were raised approximately fourfold (clamp $\mathrm{G}$ and clamp I, respectively) to achieve the same rate of glucose uptake as the control clamp $(P=\mathrm{NS})$.

$\mathrm{mmol} /$ liter of G6P. This is believed to be a sensitive indicator of changes in glycogen synthase activity in vivo (41).

Statistical analysis. All data are expressed as mean \pm SEM. Data calculations and statistical analysis were performed using the StatView program (BrainPower Inc., Calabasas, CA). Statistical significance was tested using analysis of variance followed by Student's two-tailed $t$ test.

\section{Results}

Rates of glucose uptake. Fig. 2 illustrates the rates of glucose uptake (GU) in the control and NIDDM subjects under basal and clamp conditions. Basal GU was $49 \%$ greater in the NIDDM subjects $(2.52 \pm 0.09$ vs. $3.75 \pm 0.23 \mathrm{mg} / \mathrm{kg}$ FFM per min, $P<0.005)$. During the clamp in the control subjects, GU was increased to $7.62 \pm 0.62 \mathrm{mg} / \mathrm{kg}$ FFM per min by raising the serum insulin level to $309 \mathrm{pmol} / \mathrm{liter}$ while clamping the glucose concentration at basal levels of $5.1 \mathrm{mmol} /$ liter (Table III). To achieve the same glucose uptake rate in the NIDDM subjects as the control subjects required plasma glucose levels to be raised an additional $\sim 15 \mathrm{mmol} /$ liter (in clamp G) or insulin raised $\sim 1,000 \mathrm{pmol} /$ liter (in clamp I) above the levels used in the control subjects (Table III). This produced glucose uptake rates of $8.62 \pm 0.49$ (clamp G) and $8.59 \pm 0.51$ (clamp I) in the NIDDM subjects (both $P=$ NS compared to controls).

Rates of glucose oxidation. Although basal glucose uptake in the NIDDM subjects was greater than the controls, basal glucose oxidation $\left(\mathrm{G}_{\mathrm{ox}}\right)$ was $28 \%$ less in the NIDDM subjects $(1.83 \pm 0.11$ vs. $1.31 \pm 0.13 \mathrm{mg} / \mathrm{kg}$ FFM per min, $P<0.005)$. This defect in $G_{o x}$ is shown in Fig. 3. Similarly, $G_{o x}$ during the clamps was $26-27 \%$ less in the NIDDM subjects under both hyperglycemic (clamp G) conditions $(4.35 \pm 0.21$ vs. $3.24 \pm 0.24, P<0.025)$ and hyperinsulinemic (clamp I) conditions (4.35 \pm 0.21 vs. $3.18 \pm 0.25, P<0.005)$ (Fig. 3).

Table III. Serum Glucose and Insulin Concentrations and Rates of Glucose Uptake in the Control and NIDDM Subjects

\begin{tabular}{lccc}
\hline & Serum glucose & Serum insulin & Glucose uptake \\
\hline & mmol/liter & pmol/liter & $m g / k g$ FFM per min \\
Control subjects & $5.1 \pm 0.1$ & $309 \pm 43$ & $7.62 \pm 0.62$ \\
NIDDM subjects & & & \\
Clamp G & $20.7 \pm 1.6^{*}$ & $258 \pm 57$ & $8.62 \pm 0.49$ \\
Clamp I & $5.0 \pm 0.1$ & $1371 \pm 388^{\ddagger}$ & $8.59 \pm 0.51$ \\
\hline
\end{tabular}

For NIDDM vs. control results: ${ }^{*} P<0.005$ and ${ }^{\ddagger} P<0.05$.
Figure 3. Rates of glucose oxidation $\left(\mathrm{G}_{\mathrm{ox}}\right)$ in the control and NIDDM subjects under basal and clamp conditions. Glucose oxidation in the NIDDM subjects was $28 \%$ lower under basal conditions $\left({ }^{* * * *} P\right.$ $<0.005$ ), $26 \%$ lower under hyperglycemic clamp conditions (clamp G, $* * P$ $<0.025$ ), and $27 \%$ lower under hyperinsulinemic clamp conditions (clamp I, **** $P<0.005$ )

Therefore, under basal conditions and both clamp conditions, irrespective of whether glucose uptake was stimulated by either hyperglycemia or hyperinsulinemia, glucose oxidation was reduced by $26-28 \%$ in the NIDDM subjects.

Rates of fat oxidation. As illustrated in Fig. 4 the rate of basal fat oxidation $\left(F_{o x}\right)$ in the control and NIDDM subjects was the same $(1.09 \pm 0.09$ vs. $0.99 \pm 0.06 \mathrm{mg} / \mathrm{kg}$ FFM per min respectively, $P=$ NS). Similarly during the clamps, although there was a trend for fat oxidation to be higher (less suppressed) in NIDD subjects, the differences were not significant $(0.16 \pm 0.08$ for the control clamp vs. $0.28 \pm 0.10$ for clamp $G$ and $0.29 \pm 0.05$ for clamp I, $P=$ NS compared to the control clamp) (Fig. 4). Free fatty acids (FFA) concentrations were also similar under basal conditions $(0.46 \pm 0.04$ vs. $0.35 \pm 0.02$ $\mathrm{mmol} /$ liter in control and NIDDM subjects, respectively, $P$ $=$ NS $)$ and during the clamps $(0.24 \pm 0.02$ in the control clamp vs. $0.22 \pm 0.04$ in clamp $G$ and $0.19 \pm 0.02$ in clamp $I, P=$ NS compared to the control clamp, data not illustrated).

Rates of nonoxidative glucose metabolism. Rates of nonoxidative glucose metabolism $\left(\mathrm{N}_{\mathrm{ox}}\right)$ in control and NIDDM subjects in the basal state and during the glucose clamps are shown in Fig. 5. Given the lower glucose oxidation and higher glucose uptake rates in the NIDDM subjects under basal conditions, it is not surprising that the basal rate of $\mathrm{N}_{\mathrm{ox}}$ was more than three times greater than the control subjects $(0.68 \pm 0.16$ vs. $2.41 \pm 0.27 \mathrm{mg} / \mathrm{kg}$ FFM per $\min , P<0.005)$. $\mathrm{N}_{\mathrm{ox}}$ was also $63-65 \%$ higher during the clamps in NIDDM subjects (3.26 \pm 0.62 in the control clamp vs. $5.32 \pm 0.54$ for clamp G and 5.38 \pm 0.68 in clamp I, $P<0.025$ and $P<0.01$ compared to the control clamp, respectively).

Glycogen synthase activity. To determine the contribution of glycogen synthesis to nonoxidative glucose metabolism we

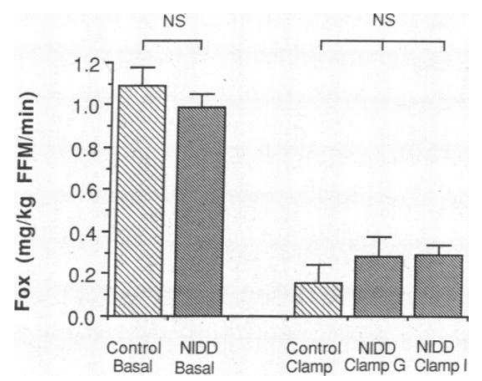

Figure 4. Rates of fat oxidation $\left(F_{o x}\right)$ in the control and NIDDM subjects under basal and clamp conditions. Fat oxidation was the same under basal conditions $(P=\mathrm{NS})$ and the same under all three clamp conditions $(P=N S)$ in the NIDDM and control subjects. 


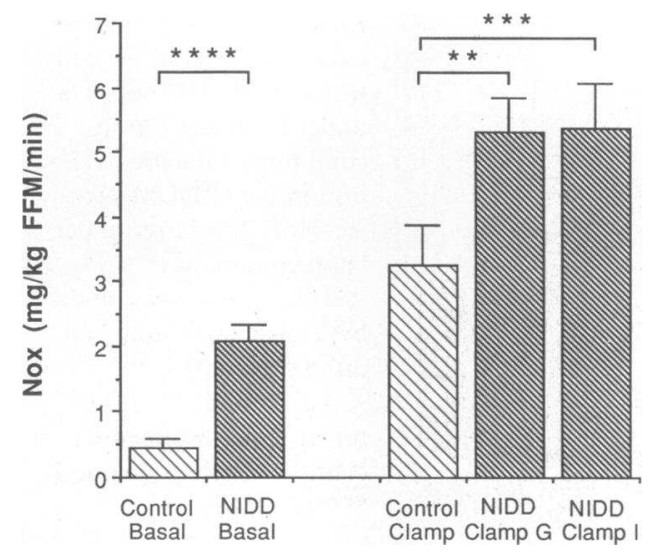

Figure 5. Rates of nonoxidative glucose metabolism $\left(\mathrm{N}_{\mathrm{ox}}\right)$ in the control and NIDDM subjects under basal and clamp conditions. Nonoxidative glucose metabolism in the NIDDM subjects was over three times higher under basal conditions $\left({ }^{* * * *} P<0.005\right), 63 \%$ higher under hyperglycemic clamp conditions (clamp G, ${ }^{* *} P$ $<0.025$ ), and $65 \%$ higher under hyperinsulinemic clamp conditions (clamp I, ${ }^{* * *} P<0.01$ ).

measured glycogen synthase activity in muscle since this enzyme is assumed to determine the amount of glycogen stored in muscle (42) and glycogen synthesis is believed to be the major component of $\mathrm{N}_{\mathrm{ox}}$ (43). It can be seen from Fig. 6 that under basal conditions glycogen synthase activity $\left(\mathrm{FV}_{0.1}\right)$ in the NIDDM subjects was $60 \%$ lower than the control subjects $(0.096 \pm 0.027$ vs. $0.038 \pm 0.007, P<0.05)$ despite the fact that the basal rates of $\mathrm{N}_{\mathrm{ox}}$ were much higher (Fig. 5). During the clamps, glycogen synthase activity in the NIDDM subjects was $41 \%$ less than the control group $(0.142 \pm 0.023$ vs. $0.084 \pm 0.012, P<0.025)$ when glucose uptake was stimulated by hyperglycemia. However, when glucose uptake was stimulated by hyperinsulinemia in the NIDDM subjects, glycogen synthase activity was the same as control subjects $(0.142 \pm 0.023$ vs. $0.130 \pm 0.027, P=N S)$. Within the NIDDM group, the lower insulin concentration in clamp $\mathrm{G}$ corresponded to significantly lower glycogen synthase activity compared to clamp I $(P<0.05)$. Therefore, the increases observed in non-oxidative glucose metabolism in the NIDDM compared to control subjects (Fig. 5) were not accompanied by corresponding increases in glycogen synthase activity (Fig. 6). In fact, when insulin levels were the same, glycogen synthase

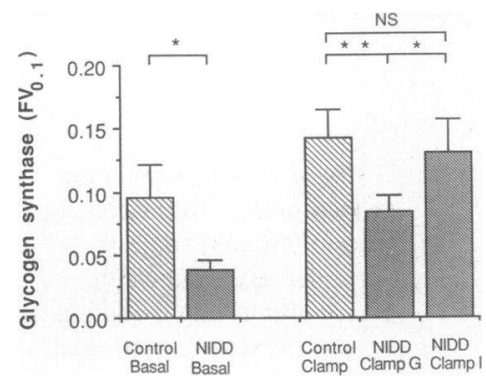

Figure 6. Rates of glycogen synthase activity expressed as fractional velocity at 0.1 $\mathrm{mmol} /$ liter GGP $\left(\mathrm{FV}_{0.1}\right)$ in the control and NIDDM subjects under basal and clamp conditions. Glycogen synthase activity in the NIDDM subjects was $60 \%$ lower under basal conditions $\left({ }^{*} P<0.05\right)$ and $41 \%$ lower under hyperglycemic clamp conditions (clamp $G,{ }^{* *} P<$ 0.025 ). Glycogen synthase activity in the NIDDM subjects under hyperinsulinemic conditions (clamp I) was not significantly different from the control subjects $(P=\mathrm{NS})$. was lower in the NIDDM subjects and only normalized to control values when insulin levels increased approximately fourfold.

Plasma lactate concentrations. Circulating plasma lactate levels were measured to compare the amount of glucose being metabolized to lactate by nonoxidative glycolysis under basal and clamp conditions in the NIDDM and control subjects. This data is illustrated in Fig. 7. Circulating basal plasma lactate levels were $50 \%$ higher in the NIDDM compared to control subjects $(0.8 \pm 0.1$ vs. $1.2 \pm 0.1, P<0.01)$. When glucose uptake was stimulated by hyperglycemia in the NIDDM subjects (clamp G), lactate levels increased by $91 \%$ compared to the control clamp levels $(1.1 \pm 0.1$ vs. $2.1 \pm 0.1, P<0.005)$. When glucose uptake was stimulated by hyperinsulinemia (clamp I), lactate levels in the NIDDM subjects also increased although to a lesser extent $(36 \%, 1.1 \pm 0.1$ vs. $1.5 \pm 0.1, P$ $<0.05$ ). Within the NIDDM group lactate levels were significantly higher when glucose uptake was stimulated by hyperglycemia vs. hyperinsulinemia $(2.1 \pm 0.1$ vs. $1.5 \pm 0.1, P<0.01)$.

\section{Discussion}

Many studies have shown that rates of intracellular glucose metabolism are lower in NIDDM subjects than nondiabetic subjects $(2,3,5,6)$. However, reduced rates of glucose oxidation and nonoxidative glucose metabolism in these studies have always been accompanied by reduced rates of glucose uptake, therefore making it impossible to determine whether these reduced rates of glucose metabolism are due to intracellular defects or simply a result of less glucose entering the cell. To overcome this problem, we measured rates of intracellular glucose metabolism when stimulated rates of glucose uptake were matched in age-sex and weight-matched NIDDM and nondiabetic control subjects. This was achieved by either raising the serum glucose or insulin concentration in the NIDDM subjects fourfold higher than the control subjects. Our results clearly show that rates of glucose oxidation are dramatically

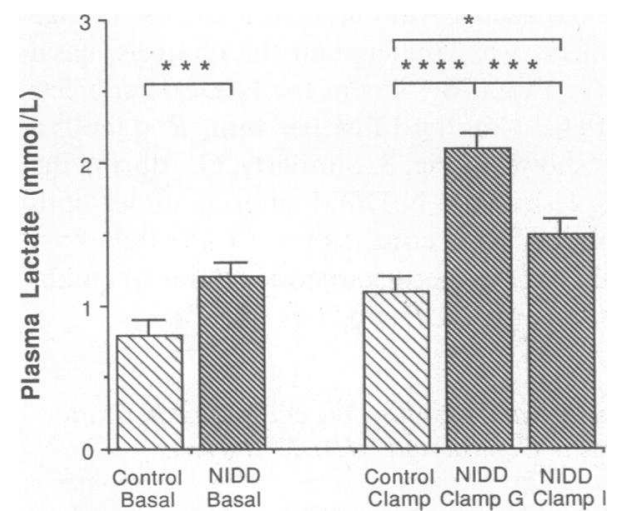

Figure 7. Plasma lactate concentrations in the control and NIDDM subjects under basal and clamp conditions. Basal lactate levels were $50 \%$ higher in the NIDDM compared to control subjects ( ${ }^{* * *} P$ $<0.01$ ). Lactate levels in the NIDDM subjects were $91 \%$ higher under hyperglycemic conditions $\left({ }^{* * * *} P<0.005\right)$ and $36 \%$ higher under hyperinsulinemic conditions $\left({ }^{*} P<0.05\right)$ compared to nondiabetic subjects. Within the NIDDM subjects, lactate levels were higher under hyperglycemic compared to hyperinsulinemic conditions $\left(^{* * *}\right.$, $P<0.01)$. L, liter. 
reduced in nonobese NIDDM subjects even when the rate of whole-body glucose uptake is matched to that of control subjects. Furthermore, the magnitude of this defect is the same whether glucose uptake is stimulated by either hyperglycemia or hyperinsulinemia, and occurs despite normal fat oxidation rates. These lower rates of glucose oxidation in NIDDM subjects are accompanied by higher rates of nonoxidative glucose metabolism when glucose uptake is matched to control subjects. Muscle glycogen synthase activity, however, is $41 \%$ lower when insulin levels are the same. Unlike glucose oxidation, this reduced glycogen synthase activity can be overcome by raising insulin concentrations fourfold.

Whole-body glucose oxidation is reduced by the same amount (26-28\%) in NIDDM compared to nondiabetic subjects under both basal and stimulated flux conditions despite glucose uptake being higher (under basal conditions) or the same (under clamp conditions). These results indicate that the defect in glucose oxidation cannot be overcome by increasing the rate of glucose uptake or the serum concentrations of glucose or insulin. Although the individual tissues responsible for this oxidative defect cannot be determined from the current studies, available evidence indicates that it is most likely to occur predominantly in skeletal muscle. This conclusion is based on regional catheterization studies conducted under conditions similar to those of the current study. These catheterization studies have shown that $\sim 90 \%$ of thc glucose taken up under hyperinsulinemic euglycemic conditions (e.g., clamp I and control study) is disposed of by muscle $(1,44)$. Similarly, under hyperglycemic conditions (e.g., in clamp G) the vast majority $(80 \%)$ of glucose taken up is disposed of by muscle (44). Even if this $10 \%$ difference in muscle glucose uptake under hyperglycemic (clamp G) or hyperinsulinemic (control clamp) conditions were taken up by tissues with no oxidative capacity, this cannot explain the $26 \%$ difference observed in glucose oxidation between the NIDDM and control subjects. Based on this line of reasoning, it seems likely that the oxidative defect in glucose metabolism occurs predominantly in skeletal muscle tissue.

Although our study was not designed to locate the metabolic abnormality which underlies the defect in glucose oxidation in NIDDM, some clues as to where the defect does not occur can be determined from our data. Impaired fat oxidation was not responsible for the defect in glucose oxidation since rates of fat oxidation were identical in the NIDDM and control subjects under both basal and stimulated rates of glucose uptake. The similarity in fat oxidation rates and free fatty acid levels in control and NIDDM subjects despite reduced rates of glucose oxidation in the NIDDM subjects in our study is supported by the findings of a number of researchers who have shown that fat metabolism in non-obese NIDDM subjects is normal despite impaired insulin-mediated glucose utilization (3-5, 45-47). Therefore, in nonobese NIDDM subjects, the defect in glucose oxidation is not associated with increased fat oxidation. The situation in NIDDM subjects who are also obese may differ, however, since a disturbance in fat metabolism in these subjects may cause an additional defect in glucose oxidation $(21,22)$.

Defects in glucose uptake were also not responsible for reduced glucose oxidation in the NIDDM subjects since reduced glucose oxidation occurred when rates of glucose uptake were normalized. This data strongly supports the notion that glucose oxidation does not regulate the rate of glucose uptake in nonobese NIDDM individuals under either hyperglycemic or hyperinsulinemic conditions.

The likely cause of reduced glucose oxidation in NIDDM is a defect in the activity of the pyruvate dehydrogenase (PDH) complex. There is some published data which has shown that a defect in PDH activity exists in NIDDM which cannot be overcome by increasing the ambient insulin concentration $(48-50)$. This would explain why no improvement in glucose oxidation was observed in the present study when insulin concentrations were increased fourfold in the NIDDM subjects. Further studies, particularly those directed at PDH activity, will be needed to define the exact cause of reduced glucose oxidation in NIDDM.

In addition to defects in oxidative glucose metabolism, defects in total nonoxidative glucose metabolism have been reported in NIDDM $(2-7,15)$. The close correlations found between decreases in nonoxidative glucose metabolism and decreases in insulin-mediated glucose uptake (2-10) have lead several investigators to speculate that defects in nonoxidative glucose metabolism may be the cause of reduced rates of glucose uptake in NIDDM and other states of insulin resistance $(7,10-15)$. However, the reduced non-oxidative glucose metabolism could simply be a result of reduced glucose uptake rather than an independent defect in NIDDM. We were able to address this issue in the present study by matching rates of glucose uptake in NIDDM and nondiabetic subjects. When glucose uptake rates were identical in NIDDM and control subjects the decreases in nonoxidative glucose metabolism which others have reported in NIDDM $(2-7,15)$ did not exist. In fact, the amount of glucose entering nonoxidative metabolism in the NIDDM subjects was $63-65 \%$ greater than the control subjects when glucose uptake was normalized using either hyperglycemia or hyperinsulinemia. These data suggest that insulin has no intracellular effect on the absolute rate of nonoxidative glucose metabolism. Since total nonoxidative glucose metabolism was greater in the NIDDM subjects when glucose uptake was the same as the control subjects, this pathway of glucose metabolism appears to serve simply as a reservoir for glucose which enters the cell and cannot be oxidized rather than a regulator of glucose uptake. Therefore, in contrast to the speculation recently raised concerning a rate-limiting role for nonoxidative glucose metabolism on glucose uptake $(7,10-15)$, our data strongly suggest that total nonoxidative glucose metabolism, like glucose oxidation, plays no role in determining the rate of glucose uptake under physiologic conditions of hyperglycemia and hyperinsulinemia.

Nonoxidative glucose metabolism may be composed of glycogen and lipid synthesis, nonoxidative glycolysis to lactate, and the pentose phosphate shunt. Of these components only glycogen synthesis and lactate formation are likely to be significant under our study conditions since no net lipogenesis (respiratory quotient $>1.0[8]$ ) was detected and the pentose phosphate shunt is negligible in muscle tissue (51). To further characterize the composition of nonoxidative glucose metabolism, muscle glycogen synthase activity was measured since this enzyme is assumed to be the major determinant of glycogen deposition in muscle $(7,42)$. Although the rate of nonoxidative glucose metabolism was greater in the NIDDM subjects, the activity of glycogen synthase was lower under basal conditions and during the clamps when insulin levels were the same. This was a surprising finding since glycogen synthesis is thought to be the major component of nonoxidative glucose 
metabolism (43). Unlike previous studies which have shown reduced glycogen synthase activity under conditions of reduced glucose uptake and reduced nonoxidative glucose metabolism $(7,10)$, our study is the first to demonstrate that this reduced activity occurs independent of changes in glucose uptake and in the presence of increased total nonoxidative glucose metabolism. In support of our findings, Wright et al. (52) have shown that 90 minutes after a meal, when insulin concentrations are the same but glucose concentrations are significantly higher (like clamp G), muscle glycogen synthase activity is lower in NIDDM compared to control subjects. In the present study, raising the insulin level approximately fourfold in the NIDDM subjects (clamp I) normalized the glycogen synthase activity to that of the control group. These data suggest that the sensitivity of glycogen synthase to insulin is lower in NIDDM compared with control subjects. Since this occurs independent of changes in glucose uptake, insulin rather than glucose availability appears to be the primary regulator of glycogen synthase in NIDDM.

Since glucose uptake was comparably stimulated, glucose oxidation comparably defective, and glycogen synthesis was not increased during the clamps in the NIDDM subjects, one would expect the remaining glucose taken up to enter the only other intracellular pathway available, namely nonoxidative glycolysis to lactate. The lactate data collected in the present study shows this to be the case. Circulating plasma lactate levels were significantly higher during both clamps in the NIDDM subjects than in control subjects. Given that less glucose enters glycogen synthesis in hyperglycemic clamp $G$ than hyperinsulinemic clamp I in the NIDDM subjects it also seems reasonable that lactate levels be significantly higher in clamp $G$ than clamp I, as our data has shown. Under basal conditions, the increase in nonoxidative glucose metabolism was also accompanied by increases in plasma lactate levels in the NIDDM compared to control subjects, as previously reported by other investigators (53-55). Thus, the mechanisms of increased nonoxidative glucose metabolism under both basal and stimulated conditions in NIDDM is not into glycogen synthesis but primarily into lactate.

In summary, the results of this study indicate that a defect in the oxidative pathway results in reduced glucose oxidation in nonobese NIDDM subjects which is independent of fat oxidation or impaired glucose uptake. A decrease in the sensitivity of glycogen synthase to insulin also appears to exist in NIDDM which is independent of insulin's effect on glucose uptake. However, this defect, unlike glucose oxidation, can be overcome by increasing the insulin concentration. The findings that glucose oxidation and glycogen synthase activity are reduced and nonoxidative glucose metabolism is increased in NIDDM subjects when rates of glucose uptake are normalized to nondiabetic rates suggests that some step proximal to the intracellular metabolism of G6P is rate-limiting for overall glucose uptake.

\section{Acknowledgments}

We gratefully acknowledge the help of Ginger Brechtel, Therese Flynn, Michelle Goldberg, and the nursing staff of the Division of Endocrinology and Metabolism; Cleon Tate for his excellent secretarial assistance; and especially the research volunteers. We thank Eli Lilly and Co., Indianapolis, IN, for kindly donating the crystalline human insulin (Humulin R).
This study was supported in part by the American Diabetes Association (ADA), the Medical Research Service of the Veterans Administration Medical Center, grant DK-38949 from the NIDDK and grant PHS RR-00827 from the General Clinical Research Branch, Division of Research Resources, National Institutes of Health. Barry Gumbiner is the recipient of an ADA California Affiliate Fellowship Grant. Anne Thorburn is a Neil Hamilton Fairley Fellow funded by National Health and Medical Research Council of Australia.

\section{References}

1. DeFronzo, R. A., R. Gunnarsson, O. Björkman, M. Olsson, and J. Wahren. 1985. Effects of insulin on peripheral and splanchnic glucose metabolism in non-insulin-dependent (Type II) diabetes mellitus. J. Clin. Invest. 76:149-155.

2. Boden, G., T. K. Ray, R. H. Smith, and O. E. Owen. 1983. Carbohydrate oxidation and storage in obese non-insulin-dependent diabetic patients: effects of improving glycemic control. Diabetes. 32:982-987.

3. Golay, A., R. A. DeFronzo, E. Ferrannini, D. C. Simonson, D. Thorin, K. Acheson, D. Thiébaud, B. Curchod, E. Jéquier, and J.-P. Felber. 1988. Oxidative and non-oxidative glucose metabolism in non-obese type II (non-insulin-dependent) diabetic patients. Diabetologia. 31:585-591.

4. Golay, A., J.-P. Felber, E. Jéquier, R. A. DeFronzo, and E. Ferrannini. 1988. Metabolic basis of obesity and noninsulin-dependent diabetes mellitus. Diabetes Metab. Rev. 4:727-747.

5. Meyer, H. U., B. Curchod, E. Maeder, P. Pahud, E. Jéquier, and J.-P. Felber. 1980. Modifications of glucose storage and oxidation in nonobese diabetics, measured by continuous indirect calorimetry. Diabetes. 29:752-756.

6. Felber, J.-P., E. Ferrannini, A. Golay, H. U. Meyer, D. Thiebaud, B. Curchod, E. Maeder, E. Jéquier, and R. A. DeFronzo. 1987 Role of lipid oxidation in pathogenesis of insulin resistance of obesity and type II diabetes. Diabetes. 36:1341-1350.

7. Bogardus, C., S. Lillioja, K. Stone, and D. Mott. 1984. Correlation between muscle glycogen synthase activity and in vivo insulin action in man. J. Clin. Invest. 73:1185-1190.

8. Lillioja, S., C. Bogardus, D. M. Mott, A. L. Kennedy, W. C. Knowler, and B. V. Howard. 1985. Relationship between insulin-mediated glucose disposal and lipid metabolism in man. J. Clin. Invest. 75:1106-1115.

9. Lillioja, S., D. M. Mott, J. K. Zawadzki, A. A. Young, W. G. Abbott, and C. Bogardus. 1986. Glucose storage is a major determinant of in vivo "insulin resistance" in subjects with normal glucose tolerance. J. Clin. Endocrinol. Metab. 62:922-927.

10. Bogardus, C., P. Thuillez, E. Ravussin, B. Vasquez, M. Narimiga, and S. Azhar. 1983. Effect of muscle glycogen depletion on in vivo insulin action in man. J. Clin. Invest. 72:1605-1610.

11. Yki-Jarvinen, H., D. Mott, A. A. Young, K. Stone, and C. Bogardus. 1987. Regulation of glycogen synthase and phosphorylase activities by glucose and insulin in human skeletal muscle. J. Clin. Invest. 80:95-100.

12. Young, A. A., C. Bogardus, K. Stone, and D. M. Mott. 1988. Insulin response of components of whole-body and muscle carbohydrate metabolism in humans. Am. J. Physiol. 254:E231-E236.

13. Mott, D. M., S. Lillioja, and C. Bogardus. 1986. Overnutrition induced decrease in insulin action for glucose storage: in vivo and in vitro in man. Metab. Clin. Exp. 35:160-165.

14. Young, A. A., C. Bogardus, D. Wolfe-Lopez, and D. M. Mott. 1988. Muscle glycogen synthesis and disposition of infused glucose in humans with reduced rates of insulin-mediated carbohydrate storage. Diabetes. 37:303-308.

15. Felber, J.-P., A. Golay, C. Felley, and E. Jéquier. 1988. Regulation of glucose storage in obesity and diabetes: metabolic aspects. Diabetes Metab. Rev. 4:691-700.

16. Olefsky, J. M. 1976. Insulin's effect on glucose oxidation inde- 
pendent of glucose transport. Biochem. Biophys. Res. Commun. 71:106-113.

17. Podskalny, J., and C. R. Kahn. 1986. Insulin activation of glycogen synthase in cultured human fibroblasts is not mediated solely via the insulin receptor. Horm. Metab. Res. 18:335-340.

18. Danforth, W. H. 1965. Glycogen synthetase activity in skeletal muscle: interconversion of two forms and control of glycogen synthesis. J. Biol. Chem. 240:588-593.

19. Larner, J., and C. Villar-Palasi. 1971. Glycogen synthase and its control. Curr. Top. Cell. Regul. 3:195-236.

20. Le Marchand-Brustel, Y., and P. Freychet. 1979. Effect of fasting and streptozotocin diabetes on insulin binding and action in the isolated mouse soleus muscle. J. Clin. Invest. 64:1505-1515.

21. Golay, A., J.-P. Felber, H. U. Meyer, B. Curchod, E. Maeder, and E. Jéquier. 1984. Study on lipid metabolism in obesity diabetes. Metab. Clin. Exp. 33:111-116.

22. Taskinen, M.-R., C. Bogardus, A. Kennedy, and B. V. Howard. 1985. Multiple disturbances of free fatty acid metabolism in noninsulin-dependent diabetes. J. Clin. Invest. 76:637-644.

23. National Diabetes Data Group. 1979. Classification and diagnosis of diabetes mellitus and other categories of glucose intolerance. Diabetes. 28:1039-1057.

24. Goldman, R. F., and E. R. Buskirk. 1961. A method for underwater weighing and the determination of body density. In Techniques for Measuring Body Composition. J. Brozek and A. Hershel, editors. National Research Council, National Academy of Sciences, Washington, DC. 78-89.

25. McGuire, E. A. H., J. H. Helderman, J. D. Tobin, R. Andres, and M. Berman. 1976. Effects of arterial versus venous sampling on analysis of glucose kinetics in man. J. Appl. Physiol. 41:565-573.

26. DeFronzo, R. A., J. D. Tobin, and R. Andres. 1979. Glucose clamp technique: a method for quantifying insulin secretion and resistance. Am. J. Physiol. 273:E214-E223.

27. Kolterman, O. G., R. S. Gray, J. Griffin, P. Burstein, J. Insel, J. A. Scarlett, and J. M. Olefsky. 1981. Receptor and post-receptor defects contribute to the insulin resistance in non-insulin-dependent diabetes mellitus. J. Clin. Invest. 68:957-969.

28. Steele, R. 1959. Influence of glucose loading and injected insulin on hepatic glucose output. Ann. N.Y. Acad. Sci. 82:420-430.

29. Chiasson, J. L., J. E. Liljenquist, W. W. Lacy, A. S. Jennings, and A. D. Cherrington. 1977. Gluconeogenesis: methodological approaches in vivo. Fed. Proc. 36:229-235.

30. Finegood, D. T., R. N. Bergman, and M. Vranic. 1987. Estimation of endogenous glucose production during hyperinsulinemiceuglycemic clamps: comparison of unlabeled and labeled exogenous glucose infusates. Diabetes. 36:914-924.

31. Molina, J. M., A. D. Baron, S. V. Edelman, and J. M. Olefsky. 1988. Variable tracer infusion rate improves determination of glucose turnover in man. Clin. Res. 36:151A. (Abstr.)

32. Jéquier, E., K. Acheson, and Y. Schutz. 1987. Assessment of energy expenditure and fuel utilization in man. Annu. Rev. Nutr. 7:187-208

33. Consoli, A., F. Kennedy, J. Miles, and J. Gerich. 1987. Determination of Krebs cycle metabolic carbon exchange in vivo and its use to estimate the individual contributions of gluconeogenesis and glycogenolysis to overall glucose output in man. J. Clin. Invest. 80:13031310.

34. Ferrannini, E. 1988. The theoretical bases of indirect calorimetry: a review. Metab. Clin. Exp. 37:287-301.

35. Desbuquois, B., and G. A. Aurbach. 1971. Use of polyethylene glycol to separate free and antibody bound peptide hormones in radioimmunoassay. J. Clin. Endocrinol. Metab. 33:732-738.

36. Itaya, K., and M. Vi. 1965. Colorimetric determination of free fatty acids in biological fluids. J. Lipid Res. 6:16-20.
37. Bergstrom, J. 1962. Muscle electrolytes in man. Scand. J. Clin. Lab. Invest. 14(Suppl. 68):7-101.

38. Nuttall, F. Q., J. Barbosa, and M. C. Gannon. The glycogen synthase system in skeletal muscle of normal humans and patients with myotonic dystrophy: effect of glucose and insulin administration. Metab. Clin. Exp. 23:561-568.

39. Thomas, J. A., K. K. Schlender, and J. Larner. 1968. A rapid filter paper assay for UDP glucose-glycogen glucosyltransferase, including an improved biosynthesis of UDP- ${ }^{14} \mathrm{C}$-glucose. Anal. Biochem. 25:486-499.

40. Lowry, O. H., N. J. Rosebrough, A. L. Farr, and R. J. Randall. 1951. Protein measurement with the Folin phenol reagent. J. Biol. Chem. 193:265-275.

41. Kochan, R. G., D. R. Lamb, E. M. Reimann, and K. K. Schlender. 1981. Modified assays to detect activation of glycogen synthase following exercise. Am. J. Physiol. 240:E197-E202.

42. Leloir, L. F., J. M. Olavarria, S. H. Goldemberg, and H. Carminatti. 1959. Biosynthesis of glycogen from uridine diphosphate glucose. Arch. Biochem. Biophys. 81:508-520.

43. Hultman, E., J. Bergström, and A. E. Roch-Nordlund. 1971. Glycogen storage in human skeletal muscle. Adv. Exp. Med. Biol. 11:273-285.

44. DeFronzo, R. A., E. Ferrannini, R. Hendler, P. Felig, and J. Wahren. 1983. Regulation of splanchnic and peripheral glucose uptake by insulin and hyperglycemia in man. Diabetes. 32:35-45.

45. Capaldo, B., R. Napoli, L. DiMarino, A. Picardi, G. Riccardi, and L. Sacca. 1988. Quantitation of forearm glucose and free fatty acid (FFA) disposal in normal subjects and Type II diabetic patients: evidence against an essential role for FFA in the pathogenesis of insulin resistance. J. Clin. Endocrinol. Metab. 67:893-898.

46. Arner, P., J. Bolinder, P. Engfeldt, and J. Östman. 1981. The antilipolytic effect of insulin in human adipose tissue in obesity, diabetes mellitus, hyperinsulinemia, and starvation. Metab. Clin. Exp. 30:753-760.

47. Swislocki, A. L. M., Y.-D. I. Chen, A. Golay, M.-O. Chang, and G. M. Reaven. 1987. Insulin suppression of plasma-free fatty acid concentration in normal individuals and patients with Type 2 (noninsulin-dependent) diabetes. Diabetologia. 30:622-626.

48. Randle, P. J., A. L. Kerbey, and J. Espinal. 1988. Mechanisms decreasing glucose oxidation in diabetes and starvation: role of lipid fuels and hormones. Diabetes Metab. Rev. 4:623-638.

49. Randle, P. J. 1986. Fuel selection in animals. Biochem. Soc. Trans. 14:799-806.

50. Mandarino, L. J., Z. Madar, O. G. Kolterman, J. M. Bell, and J. M. Olefsky. 1986. Adipocyte glycogen synthase and pyruvate dehydrogenase in obese and Type II diabetic subjects. Am. J. Physiol. 251:E489-E496.

51. Wood, T. 1986. Distribution of the pentose phosphate pathway in living organisms. Cell Biochem. Funct. 4:235-240.

52. Wright, K. S., H. Beck-Nielsen, O. G. Kolterman, and L. J. Mandarino. 1988. Decreased activation of skeletal muscle glycogen synthase by mixed-meal ingestion in NIDDM. Diabetes. 37:436-440.

53. Consoli, A., N. Nurjhan, J. Reilly, and J. Gerich. 1988. Importance of availability of lactate and efficiency of its conversion to glucose for the increased gluconeogenesis of non-insulin-dependent diabetes (NIDDM). Clin. Res. 36:151A. (Abstr.)

54. Zawadzki, J. K., R. R. Wolfe, D. M. Mott, S. Lillioja, B. V. Howard, and C. Bogardus. 1988. Increased rate of Cori cycle in obese subjects with NIDDM and effect of weight reduction. Diabetes. 37:154-159.

55. Berger, M., S. A. Hagg, M. N. Goodman, and N. B. Ruderman. 1976. Glucose metabolism in perfused skeletal muscle: effects of starvation, diabetes, fatty acids, acetoacetate, insulin and exercise on glucose uptake and disposition. Biochem. J. 158:191-202. 